\title{
Control of Constrained Linear Systems using Fast Sampling Rates
}

\author{
Juan Yuz ${ }^{\mathrm{a}, 1}$, Graham Goodwin ${ }^{\mathrm{a}}$, Arie Feuer $^{\mathrm{b}}$ \& \\ Jose De Donáa ${ }^{\mathrm{a}}$. \\ a School of Electrical Engineering and Computer Science, The University of \\ Newcastle, Callaghan NSW 2308, Australia. \\ ${ }^{\mathrm{b}}$ Department of Electrical Engineering, Technion-Israel Institute of Technology, \\ Technion City, Haifa 32000, Israel.
}

\begin{abstract}
This paper addresses the problem of optimal control of constrained linear systems when fast sampling rates are utilised. We show that there exists a well defined limit as the sampling rate increases. An immediate consequence of this result is the existence of a finite sampling period such that the achieved performance is arbitrarily close to the limiting performance.
\end{abstract}

Key words: constrained systems, fast sampling rate, optimal control.

\section{Introduction}

An increasingly common strategy to deal with constraints in control is Receding Horizon or Model Predictive Control (MPC) strategies [4,5,9,7,12,15-17]. This is a form of (discrete-time) control in which the current control action is obtained by solving on-line, at each sampling instant, a finite horizon optimal control problem for the open-loop plant using the current (observed) state as initial condition. The first component of the optimal control sequence is applied to the system, and the procedure is repeated again at the next sampling instant. One of the key advantages of these strategies is that constraints can be taken into account in the optimization procedure, for example, via Quadratic Programming (QP) [18].

$\overline{1}$ Corresponding author: jiyuze@ee.newcastle.edu.au 
Traditionally, MPC has been applied to systems having long time constants, e.g. petrochemical processes. However, recent advances in this area and increasing computer speeds have opened the door to short time constant applications including aerospace, automobile control and electromechanical servo problems. These applications typically use fast sampling rates and a common design goal is that the sample-hold nature of the input should have minimal impact on the achievable performance. This goal is consistent with the control of linear systems in the absence of constraints. Indeed, it is common for the sampling rate to be chosen so that the response of the sampled-data control system is practically indistinguishable from the corresponding continuous-time solution. The goal of constrained control will often be similar, i.e., it is desirable to choose a sampling period such that the artifacts of sampling are practically unobservable.

The analysis presented in this paper shows that there exists a finite sampling period such that the sampled-data response of a finite horizon constrained linear controller is arbitrarily close to the response which would be achieved by a continuous-time constrained linear controller. For background on constrained optimal control, the reader is referred to [2,19], for the continuous-time case, and $[4,5,9,7,12,15-17]$ for discrete-time case.

Previous work having a connection with our work has been reported in $[10,14]$. Reference [14] deals with unconstrained problems, while reference [10] considers finite horizon constrained LQR problems in continuous-time using spectral properties. In their approach, however, the constraints are satisfied only at a finite set of points over the control horizon. By way of contrast, we show that the continuous-time solution, with constraints imposed for all time, can be arbitrarily approximated using a standard discrete-time approach provided one chooses a suitably fast sampling rate and, possibly, tighter constraints at the sampling instants.

The layout of the remainder the paper is as follows: in Section 2 we present the continuous-time optimal control problem $\mathcal{P}$, and an associated sampleddata problem $\mathcal{P}_{\Delta}$. Section 3 gives some preliminary results. Section 4 presents the main result of the paper, namely that the cost achieved by solving the discrete-time sampled-data problem converges to the limiting cost achievable in a continuous-time framework. An illustrative example is presented in Section 5. Finally, in Section 6, we briefly discuss the receding horizon version of the problem. Conclusions are presented in Section 7. 


\section{Fixed Horizon Problem Specification}

Before considering the moving horizon problem, we first address the fixed horizon case. The latter problem is usually solved in discrete-time MPC strategies at each sampling instant. We consider two related problems, one defined in continuous-time and an associated discrete-time sampled-data problem.

\subsection{Continuous-time problem}

As a benchmark problem, we next consider a fixed horizon constrained control problem $\mathcal{P}$, defined in the continuous-time domain as follows:

(i) A continuous-time model:

$$
\begin{aligned}
\dot{x}(t) & =A x(t)+B u(t) \quad ; x(0)=x_{o} \\
y(t) & =C x(t)
\end{aligned}
$$

where $A \in \mathbb{R}^{n \times n}, B \in \mathbb{R}^{n \times m}, C \in \mathbb{R}^{m \times n}$.

(ii) A fixed time horizon $T_{f}<\infty$.

(iii) A quadratic cost function:

$$
J(u)=J_{1}+J_{\infty}
$$

where:

$$
\begin{aligned}
J_{1} & =\int_{0}^{T_{f}}\left(x(t)^{T} Q x(t)+u(t)^{T} R u(t)\right) \mathrm{d} t \\
J_{\infty} & =x\left(T_{f}\right)^{T} P x\left(T_{f}\right)
\end{aligned}
$$

with $Q \geq 0, R>0$, and where the final state weighting matrix, $P$, gives rise to the infinite horizon optimal unconstrained cost associated with the cost $J_{1}$ when the initial state is $x\left(T_{f}\right)$. Thus $P$ satisfies the continuoustime algebraic Riccati equation:

$$
0=Q+A^{T} P+P A-P B R^{-1} B^{T} P
$$

(iv) And a set of constraints for the state and/or the input signal, written in the following general form:

$$
\begin{aligned}
& L_{u} u(t) \leq M_{u} \\
& L_{x} x(t) \leq M_{x}
\end{aligned}
$$

for all $t \in\left[0, T_{f}\right]$. Note that $L_{u}$ will typically have $\operatorname{rank} m$ whilst $L_{x}$ will have rank less than or equal to $n$. 
Let $\mathcal{U} \subset \mathbb{R}^{m}$ and $\mathcal{X} \subset \mathbb{R}^{n}$ be the sets of all possible values of $u(t)$ and $x(t), t \in\left[0, T_{f}\right]$, such that (1) and (7) are satisfied. Then, the only requirements on $L_{u}, L_{x}, M_{u}$, and $M_{x}$ are that the sets $\mathcal{U}$ and $\mathcal{X}$ are bounded (possibly as a function of $x_{o}$ in the case of $\mathcal{X}$ ) and contain the origin of the respective space - see [6]. Note that this implies that all entries in both $M_{u}$ and $M_{x}$ are positive.

Given (i)-(iv), the continuous-time problem $\mathcal{P}$ is to find the optimal control signal $u^{*}=u^{*}(t)$ such that the cost function (3) is minimised, i.e.:

$$
u^{*}(t)=\arg \min _{u(t) \in \mathcal{U}} J(u)
$$

Remark 1 There has been substantial work, spanning three centuries, but particularly since the 1950's, on the general conditions under which optimal control problems such as $\mathcal{P}$ have a solution. These results give necessary and sufficient conditions under which a solution is truly a minimiser. Moreover, existence theorems for this minimiser assume that the problem is feasible, i.e., that the class of admissible pairs $\{u(t), x(t)\}$ which satisfy the system dynamic equation (1) and constraints (7) is non-empty. This is discussed, for example, in [2] and [19]. We will thus assume the existence of a solution of the continuous-time constrained optimal control problem $\mathcal{P}$, even though an explicit expression will not be obtained. Instead, we show that, subject to existence, the solution can be approximated to any desired degree of accuracy by solving an associated sampled-data constrained optimal control problem $\mathcal{P}_{\Delta}$ (described in the next subsection).

Remark 2 Note that it is common in Receding Horizon Control to utilise a final state weighting matrix as in (5) (see also equation (14) below) [6,9,12].

\subsection{Sampled-data problem}

A natural way to approximate the continuous-time problem $\mathcal{P}$ is to use a (small) sampling period $\Delta$ together with a Zero Order Hold $(\mathrm{ZOH})$ approximation to the input signal, that is, for a given sampling interval $\Delta$ :

$$
u(t)=u_{k} \quad ; k \Delta \leq t<k \Delta+\Delta
$$

where $k \in \mathbb{Z}$. Furthermore, we assume that the sampling interval is an integer fraction of the fixed time horizon $T_{f}$, i.e.:

$$
\Delta=\frac{T_{f}}{N} \Longleftrightarrow N \Delta=T_{f}
$$

for some $N \in \mathbb{N}$. 
We next define a discrete-time contrained optimal control problem $\mathcal{P}_{\Delta}$, which, in fact, corresponds to a sampled-data version of problem $\mathcal{P}$. We consider:

(i) A discrete-time model:

$$
\begin{aligned}
x_{k+1} & =A_{q} x_{k}+B_{q} u_{k} \quad ; x_{o} \text { as in }(1) \\
y_{k} & =C_{q} x_{k}
\end{aligned}
$$

where $k \in \mathbb{Z}$, and the system matrices are given by $[1,13]$ :

$$
A_{q}=e^{A \Delta} \quad ; \quad B_{q}=\int_{0}^{\Delta} e^{A \eta} B d \eta \quad ; \quad C_{q}=C
$$

which ensures that $x_{k}=x(k \Delta)$ and $y_{k}=y(k \Delta)$.

(ii) A fixed discrete-time horizon $N=\frac{T_{f}}{\Delta}$.

(iii) A quadratic cost function:

$$
J_{\Delta}\left(u_{k}\right)=\sum_{k=0}^{N-1}\left[x_{k}^{T} \quad u_{k}^{T}\right]\left[\begin{array}{ll}
Q_{q} & S_{q} \\
S_{q}^{T} & R_{q}
\end{array}\right]\left[\begin{array}{l}
x_{k} \\
u_{k}
\end{array}\right]+x_{N}^{T} P_{\Delta} x_{N}
$$

where:

$$
\begin{aligned}
Q_{q} & =\int_{0}^{\Delta} e^{A^{T} t} Q e^{A t} \mathrm{~d} t \\
S_{q} & =\int_{0}^{\Delta} e^{A^{T} t} Q h(t) \mathrm{d} t \\
R_{q} & =\int_{0}^{\Delta} h(t)^{T} Q h(t) \mathrm{d} t+R \Delta \\
h(t) & =\int_{0}^{t} e^{A(t-\tau)} B \mathrm{~d} \tau
\end{aligned}
$$

and where $P_{\Delta}$ satisfies the following discrete-time algebraic Riccati equation:

$$
P_{\Delta}=Q_{q}+A_{q}^{T} P_{\Delta} A_{q}-\left(A_{q}^{T} P_{\Delta} B_{q}+S_{q}\right)\left(R_{q}+B_{q}^{T} P_{\Delta} B_{q}\right)^{-1}\left(B_{q}^{T} P_{\Delta} A_{q}+S_{q}^{T}\right)
$$

(iv) And a set of discrete-time constraints for the state and/or the input signal, written in the following form:

$$
\begin{aligned}
& L_{u} u_{k} \leq \alpha(\Delta) M_{u} \\
& L_{x} x_{k} \leq \alpha(\Delta) M_{x}
\end{aligned}
$$

for all $k \in\{0, \ldots, N-1\}$ and where $\alpha(\Delta) \in(0,1]$ is a scaling factor, whose choice will be discussed in the next section.

Given (i)-(iv), the sampled-data problem $\mathcal{P}_{\Delta}$ is to find the optimal control 
sequence $u^{*}=u_{\Delta, k}^{*}$ such that the cost function (14) is minimised, i.e.:

$$
u_{\Delta, k}^{*}=\arg \min _{u_{k}} J_{\Delta}\left(u_{k}\right)
$$

Remark 3 It is well-known, by using the delta operator [8,13], that:

$$
\frac{A_{q}-I}{\Delta} \rightarrow A, \quad \frac{B_{q}}{\Delta} \rightarrow B, \quad \frac{Q_{q}}{\Delta} \rightarrow Q, \quad \frac{S_{q}}{\Delta} \rightarrow 0, \quad \frac{R_{q}}{\Delta} \rightarrow R, \quad P_{\Delta} \rightarrow P
$$

as the sampling period $\Delta$ tends to 0 . This means that the unconstrained sampled-data problem given by (i)-(iii) converges to the description of the underlying unconstrained continuous-time problem given by (i)-(iii) in Section 2.1.

We next consider the corresponding constrained case, together with additional requirements on the scaling factor $\alpha(\Delta)$ in $(27)-(29)$ (below). We show that the formulation of the sampled-data problem $\mathcal{P}_{\Delta}$ converges, as $\Delta \rightarrow 0$, to the formulation of problem $\mathcal{P}$, defined in continuous-time.

\section{Preliminary Results}

While the explicit solution of the continuous time problem $\mathcal{P}$ is very difficult to obtain, solving $\mathcal{P}_{\Delta}$ for a given sampling period $\Delta$ is relatively straightforward using standard numerical procedures, e.g., QP [9]. Our goal is to relate the solution of $\mathcal{P}_{\Delta}$ to the solution of $\mathcal{P}$.

We first introduce some definitions and notation that will be used in the sequel. For a given sampling interval $\Delta$, we define $u_{\Delta}(t)$ as a piece-wise constant continuous-time signal of the form:

$$
u_{\Delta}(t)=u_{k} \quad ; k \Delta \leq t<k \Delta+\Delta
$$

where $k \in \mathbb{Z}$.

It can be readily shown (see, for example, [1]) that the choices (15) to (19) ensure that:

$$
J\left(u_{\Delta}\right)=J_{\Delta}\left(u_{k}\right)
$$

where $J_{\Delta}\left(u_{k}\right)$ denotes the discrete-time cost value, as defined in (14), when the control sequence $\left\{u_{k}\right\}$ is applied to the system (11)-(12), and $J\left(u_{\Delta}\right)$ denotes the continuous-time cost value in $(3)-(6)$ when $u_{\Delta}(t)$, as defined in $(23)$, is applied to the continuous-time system (1)-(2). 
We next discuss the scalar $\alpha(\Delta)$ introduced earlier, in equation (20), as a scaling factor associated with the discrete-time constraints.

Given the continuous-time system (with its initial condition $x_{o}$ ) in (1), and the constraints (7), we define the following sets:

$$
S=\left\{u \in \mathcal{L}_{2}\left[0, T_{f}\right]: L_{u} u(t) \leq M_{u} \text { and } L_{x} x(t) \leq M_{x}, \forall t \in\left[0, T_{f}\right]\right\}
$$

and:

$$
\begin{aligned}
S_{\Delta}=\left\{u \in \mathcal{L}_{2}\left[0, T_{f}\right]: L_{u} u(t) \leq \alpha(\Delta) M_{u}, \forall t \in\left[0, T_{f}\right]\right. & \\
& \text { and } \left.L_{x} x(k \Delta) \leq \alpha(\Delta) M_{x}, \forall k \in\{0, \ldots, N-1\}\right\}
\end{aligned}
$$

Remark 4 Note that $S$ contains all possible signals $u(t)$ among which we need to find the one that minimises the cost function $J(u)$ in (3)-(6), i.e., $u^{*}(t)$. On the other hand, every sequence $u_{k}$ (including $u_{k}^{*}$, the solution of $\mathcal{P}_{\Delta}$ ) satisfying the difference equation (11) and the constraints (20) will generate a piece-wise signal $u_{\Delta}(t)$, as in (23), that belongs to $S_{\Delta}$.

However, even if the scaling factor $\alpha(\Delta)$ is chosen less than $1, u_{\Delta}(t) \in S_{\Delta}$ does not necessarily imply that $u_{\Delta}(t) \in S$, because of the intersample state trajectory. Thus, we next impose further requirements on the scalar $\alpha(\Delta)$.

Given a sequence $\left\{\Delta_{i}>0\right\}$, such that:

$$
\Delta_{i}>\Delta_{i+1} \quad \text { and } \quad \lim _{i \rightarrow \infty} \Delta_{i}=0
$$

we require the following properties for $\alpha\left(\Delta_{i}\right)$ and for the resulting sequence of sets $\left\{S_{\Delta_{i}}\right\}$ :

$$
\begin{array}{ll} 
& \lim _{i \rightarrow \infty} \alpha\left(\Delta_{i}\right)=\lim _{\Delta_{i} \rightarrow 0} \alpha\left(\Delta_{i}\right)=1 \\
\text { and } \quad S_{\Delta_{i}} \subseteq S_{\Delta_{i+1}}
\end{array}
$$

Condition (28) ensures that, as the sampling rate increases, the sequence of discrete-time constraints (20) approaches the continuous-time constraints in (7). Moreover, from the definitions in (25) and (26), we have that:

$$
\lim _{i \rightarrow \infty} S_{\Delta_{i}}=\lim _{\Delta_{i} \rightarrow 0} S_{\Delta_{i}}=S
$$

Furthermore, the requirement (29) ensures that the sequence of sets $\left\{S_{\Delta_{i}}\right\}$ approach the set $S$ from the interior, i.e.:

$$
S_{\Delta_{0}} \subseteq S_{\Delta_{1}} \subseteq \ldots \subseteq S
$$


A particular choice for the sequences $\left\{\Delta_{i}\right\}$ and $\left\{\alpha\left(\Delta_{i}\right)\right\}$ ensuring that the above requirements are satisfied, is described in the following result:

Lemma 5 Consider the sequence of sampling intervals $\left\{\Delta_{i}\right\}$, defined by:

$$
\Delta_{i}=\frac{2^{2-i}}{\|A\|} \ln \left(\frac{\sqrt{1+4 \gamma^{2}}}{2 \gamma}\right)
$$

Then, one particular choice for the scaling factor $\alpha(\Delta)$ that satisfies (28)-(29) is given by:

$$
\alpha(\Delta)=\frac{1}{1+(2 \gamma+1)\left(e^{\|A\| \Delta}-1\right)}
$$

where $\gamma$ is a constant given by:

$$
\gamma=\left(\max _{i} \frac{\left\|L_{x}^{i}\right\|}{M_{x}^{i}}\right)\left(X+\frac{\|B\|}{\|A\|} U\right)
$$

and where $L_{x}^{i}$ and $M_{x}^{i}$ are the $i$-th row and $i$-th entry of $L_{x}$ and $M_{x}$ respectively, and where $X$ and $U$ denote upper bounds on the norms of $x(t)$ and $u(t)$ in the bounded sets $\mathcal{X}$ and $\mathcal{U}$ respectively.

PROOF. See Appendix.

We will need two additional technical results:

Lemma 6 Let $u(t) \in S$. Then, for any $\delta>0$ there exists $a \Delta_{\delta}>0$ such that, for all $\Delta \leq \Delta_{\delta}$ and $u_{\Delta}(t)$ such that:

$$
u_{\Delta}(t)=u(k \Delta) \quad k \Delta \leq t<(k+1) \Delta
$$

we have:

$$
\left\|u-u_{\Delta}\right\|_{2}<\delta
$$

PROOF. See Appendix.

Lemma 7 For any $\varepsilon>0$ there exists a $\delta_{\varepsilon}>0$ such that if:

$$
\left\|u-u^{*}\right\|_{2}<\delta_{\varepsilon}
$$

then:

$$
J(u)-J\left(u^{*}\right)<\varepsilon
$$


PROOF. See Appendix.

Remark 8 Lemma 6 states that any input signal $u(t) \in S$ can be arbitrarily approximated, in an $\mathcal{L}_{2}$ sense, by the sample-and-hold signal $u_{\Delta}(t)$. On the other hand, Lemma 7 establishes that the optimal continuous-time performance $J\left(u^{*}\right)$ can be arbitrarily approximated by choosing any signal $u(t)$ sufficiently close, in an $\mathcal{L}_{2}$ sense, to $u^{*}(t)$. These two facts will be used in the proof of our main result in the next section.

\section{Main Result}

In this section we present our main result, namely that the optimal performance obtained by solving the continuous-time problem $\mathcal{P}$ can be arbitrarily approximated by solving the discrete-time problem $\mathcal{P}_{\Delta}$.

First we note that, for any chosen $\Delta$, the corresponding problem $\mathcal{P}_{\Delta}$ is well defined and can be solved. We denote the resulting optimal control sequence by $\left\{u_{\Delta, k}^{*}\right\}_{k=0}^{N-1}$.

We can now state our main result as:

Theorem 9 Provided $\mathcal{P}$ has a solution $u^{*}(t) \in S$, then, for every $\varepsilon>0$ there exists a $\Delta_{\varepsilon}$, such that:

$$
J\left(u_{\Delta_{\varepsilon}}^{*}\right)-J\left(u^{*}\right)<\varepsilon
$$

where $u_{\Delta_{\varepsilon}}^{*}(t)$ is generated, using the $Z O H$ (23), by the sequence $u_{\Delta_{\varepsilon}, k}^{*}$, the solution of problem $\mathcal{P}_{\Delta_{\varepsilon}}$.

PROOF. Given $\varepsilon>0$ we know by Lemma 7 that there exists a $\delta_{\varepsilon}>0$ such that for every $u \in \mathcal{L}_{2}\left[0, T_{f}\right]$ for which (37) holds, (38) also holds.

Since $u^{*} \in S$ and $\lim _{i \rightarrow \infty} S_{\Delta_{i}}=S$, there exists $\Delta_{\ell}>0$ for which the set

$$
\mathcal{A}=\left\{u \in \mathcal{L}_{2}\left[0, T_{f}\right]:\left\|u-u^{*}\right\|_{2}<\delta_{\varepsilon}\right\} \cap S_{\Delta_{\ell}}
$$

is not empty. Let $\bar{u}$ be in this set. The set $\left\{u \in \mathcal{L}_{2}\left[0, T_{f}\right]:\left\|u-u^{*}\right\|_{2}<\delta_{\varepsilon}\right\}$ is open. Hence, there exists $\delta_{1}>0$ such that:

$$
\left\{u \in \mathcal{L}_{2}\left[0, T_{f}\right]:\|u-\bar{u}\|_{2}<\delta_{1}\right\} \subset \mathcal{A}
$$

Now, using Lemma 6, we know that $\bar{u}$ can be arbitrarily approximated in an $\mathcal{L}_{2}$ sense by a piece-wise constant function. This means that there exists a 
sampling period $\Delta_{j}<\Delta_{\ell}$ such that:

$$
\left\|\bar{u}-\bar{u}_{\Delta_{j}}\right\|_{2}<\delta_{1}
$$

Hence, $\bar{u}_{\Delta_{j}} \in S_{\Delta_{\ell}} \subset S_{\Delta_{j}} \subset S$ and $\left\|\bar{u}_{\Delta_{j}}-u^{*}\right\|_{2}<\delta_{\varepsilon}$, as is represented schematically in Figure 1. By Lemma 7, the latter implies that:

$$
J\left(\bar{u}_{\Delta_{j}}\right)-J\left(u^{*}\right)<\varepsilon
$$

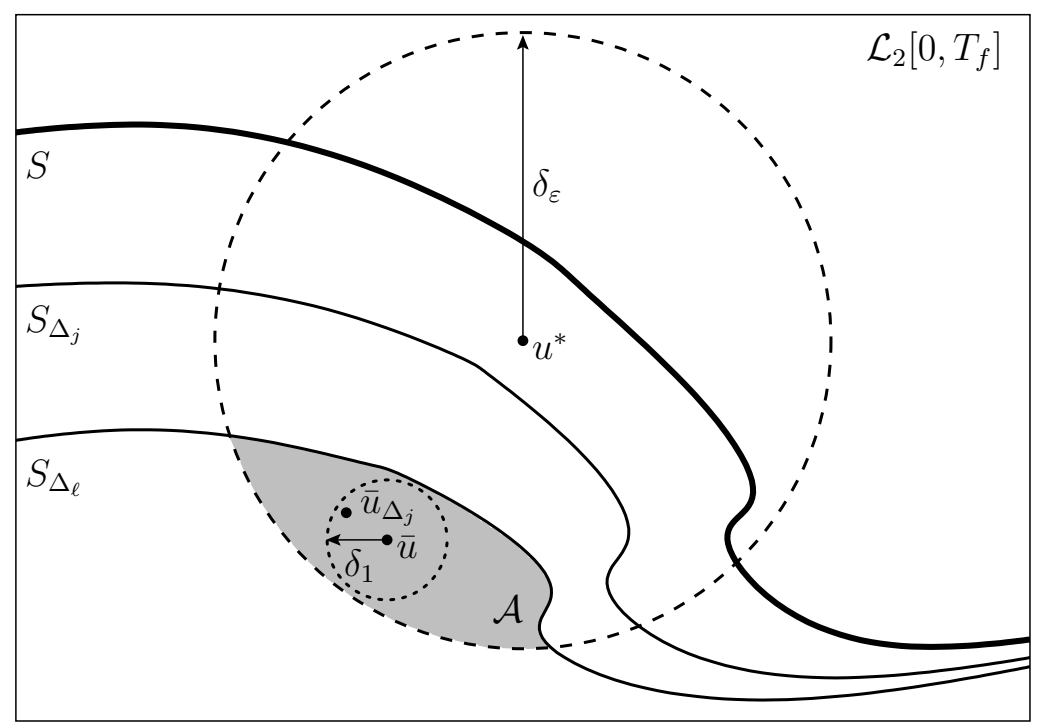

Fig. 1. Schematic representation of proof of Theorem 9.

If we now consider $u_{\Delta_{j}, k}^{*}$, the solution of the problem $\mathcal{P}_{\Delta_{j}}$, we know that:

$$
J_{\Delta}\left(\bar{u}_{\Delta_{j}, k}\right) \geq J_{\Delta}\left(u_{\Delta_{j}, k}^{*}\right)
$$

where $\bar{u}_{\Delta_{j}, k}$ is the sequence with the values taken by $\bar{u}_{\Delta_{j}}(t)$. Using (24), we then have that:

$$
J\left(\bar{u}_{\Delta_{j}}\right) \geq J\left(u_{\Delta_{j}}^{*}\right)
$$

and equation (39) follows by choosing $\Delta_{\varepsilon}=\Delta_{j}$. This completes the proof of the theorem.

The theorem above establishes our main claim in this paper, namely, that there exists a finite sampling period $\Delta$, such that the performance achieved with the optimal fixed horizon discrete-time constrained controller is as close as desired to the performance achievable by the optimal fixed horizon continuous-time constrained controller. 


\section{Example}

We illustrate the results in Section 3 by a simple example:

Example 10 Consider the second order system:

$$
\begin{aligned}
& \dot{x}=\left[\begin{array}{cc}
-2 & 0 \\
1 & -1
\end{array}\right] x+\left[\begin{array}{l}
4 \\
0
\end{array}\right] u \quad ; x_{o}=\left[\begin{array}{l}
0 \\
2
\end{array}\right] \\
& y=\left[\begin{array}{ll}
0 & 1
\end{array}\right] x
\end{aligned}
$$

with continuous cost function (3), where $T_{f}=5, Q=C^{T} C, R=0.1$, and where $P$ is the solution of the algebraic Riccati equation (6). We impose constraints on the input and on one state as follows:

$$
\begin{aligned}
|u(t)| \leq 1 & \Longleftrightarrow\left[\begin{array}{c}
1 \\
-1
\end{array}\right] u(t) \leq\left[\begin{array}{l}
1 \\
1
\end{array}\right] \\
\left|x_{1}(t)\right| \leq 1 & \Longleftrightarrow\left[\begin{array}{cc}
1 & 0 \\
-1 & 0
\end{array}\right] x(t) \leq\left[\begin{array}{l}
1 \\
1
\end{array}\right]
\end{aligned}
$$

The matrices of the discrete-time sampled model (11)-(12) are obtained from equation (13). Similarly, the matrices for the sampled-data cost function (14), are obtained from equations (15)-(19).

Figure 2 shows the evolution of the cost function as $N$ increases (i.e. $\Delta$ decreases), for the constrained and unconstrained cases. Note that a logarithmic scale has been used. For the unconstrained case, it can be seen that the value of the cost function approaches the continuous time optimal result. Similarly, for the constrained case, we can see that beyond $N=16$, the minimum achievable for the cost function is almost constant. This is also confirmed in Figure 3, which shows the convergence of the (ZOH or piece-wise constant) control and state signals for the constrained case.

\section{Receding Horizon Problem}

We finally make some brief comments on the moving horizon control problem. An issue here is that usually, in discrete receding horizon strategies, only the first element of the fixed horizon control solution is applied at each step. The problem is then solved again at the next sampling instant. 


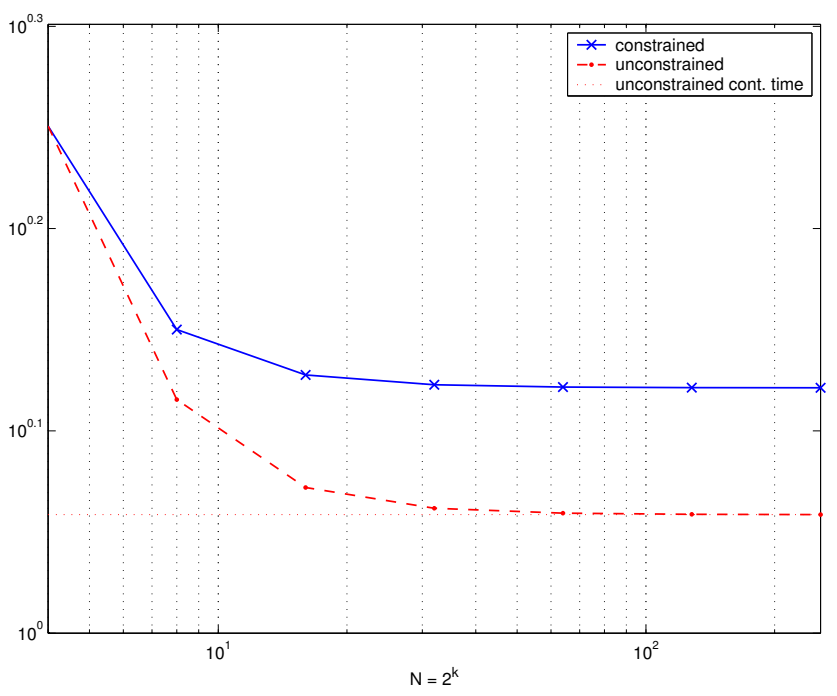

Fig. 2. Cost function values $\mathrm{v} / \mathrm{s} N$
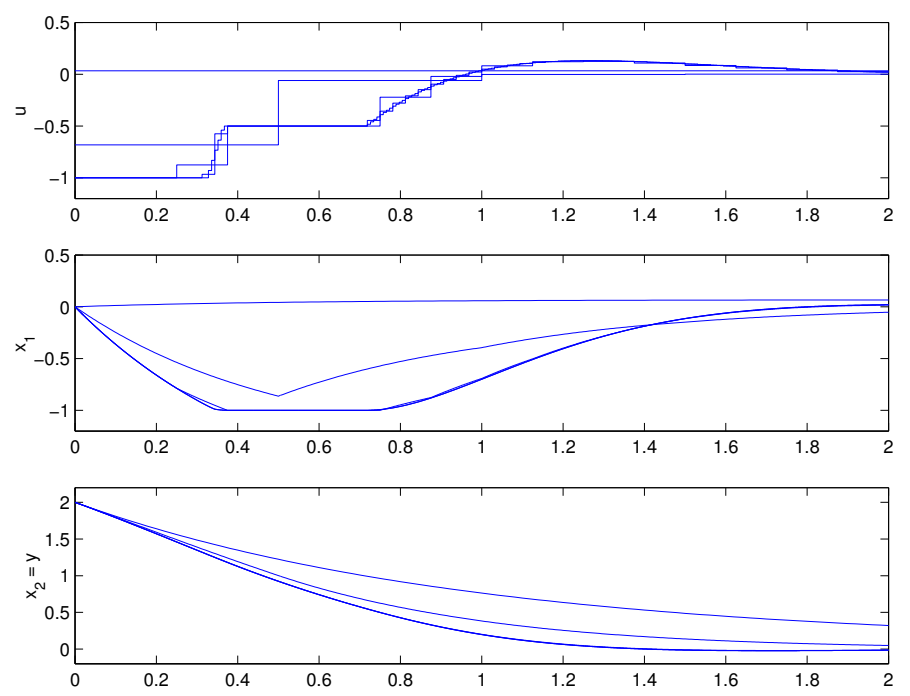

Fig. 3. Control and state signals for $N=1,4, \ldots, 256$

If we take the limit of this procedure as $\Delta$ tends to zero, we end up with an ill-defined control law because the optimal continuous-time solution is only unique up to an $\mathcal{L}_{2}$ equivalence.

To address this issue, we will adopt a form of discrete MPC which mirrors the common strategy suggested for continuous-time predictive control [3], namely, the control input to the plant is changed every $\Delta$ (seconds), but the fixed horizon optimization is done only every $\bar{\Delta}$ (seconds), where $\bar{\Delta}>\Delta$.

Let $\ell$ be an integer, and define:

$$
u_{F H}^{*}(\ell \bar{\Delta}, \tau)=u^{*}(\tau) \quad \forall \tau \in[0, \bar{\Delta}[
$$


where $u^{*}(\tau)$ is the solution to the fixed horizon constrained continuous-time problem on the interval $\tau \in\left[0, T_{f}\right]$.

We then define the continuous time moving horizon optimal solution in terms of (50) as:

$$
u_{M H}^{*}(t)=u_{F H}^{*}(\ell \bar{\Delta}, t-\ell \bar{\Delta}) \quad \forall t \in[\ell \bar{\Delta},(\ell+1) \bar{\Delta}[
$$

We then have the following result:

Theorem 11 . The discrete-time approximation of the receding horizon strategy defined above, where the control is restricted to be piece-wise constant over every interval $[k \Delta,(k+1) \Delta)$, converges to the continuous-time result, i.e.:

$$
\lim _{\Delta \rightarrow 0}\left\|u_{M H \Delta}^{*}(t)-u_{M H}^{*}(t)\right\|_{2}=0
$$

PROOF. The result follows immediately from the definitions of $u_{M H}^{*}$ in (51) and (50), and the previous convergence result in Theorem 9

\section{Conclusions}

This paper has explored the use of fast sampling rates in the control of constrained linear systems. We have shown that, as the sampling period approaches zero, the optimal continuous-time problem can be approximated arbitrarily close by a corresponding discrete-time sampled-data problem.

\section{A Appendix}

Proof of Lemma 5

We show that the choice of $\left\{\Delta_{i}\right\}$ and $\alpha(\Delta)$ given in (32)-(34), satisfies the requirements (27)-(29).

With $\Delta_{i}$ as in (32) we clearly have (27), and, as a consequence, $\alpha(\Delta)$ in (33) satisfies (28).

To show that (29) holds, let us take any $u \in S_{\Delta_{i}}$, which by definition (26) 
inplies that:

$$
L_{u} u(t) \leq \alpha\left(\Delta_{i}\right) M_{u} \quad \text { and } \quad L_{x} x\left(k \Delta_{i}\right) \leq \alpha\left(\Delta_{i}\right) M_{x}
$$

Since $\alpha\left(\Delta_{i}\right)<\alpha\left(\Delta_{i+1}\right)$ we have that $L_{u} u(t) \leq \alpha\left(\Delta_{i+1}\right) M_{u}$ (recall that the entries of $M_{u}$ are positive). Furthermore, solving the differential equation (1) for $t=\sigma+k \Delta_{i}, 0 \leq \sigma<\Delta_{i}$, we have:

$$
\begin{aligned}
L_{x} x(t)= & L_{x}\left(e^{A \sigma} x\left(k \Delta_{i}\right)+\int_{0}^{\sigma} e^{A(\sigma-\tau)} B u\left(\tau+k \Delta_{i}\right) \mathrm{d} \tau\right) \\
= & L_{x} x\left(k \Delta_{i}\right)+L_{x}\left(\left(e^{A \sigma}-I\right) x\left(k \Delta_{i}\right)+\int_{0}^{\sigma} e^{A(\sigma-\tau)} B u\left(\tau+k \Delta_{i}\right) \mathrm{d} \tau\right) \\
\leq & \alpha\left(\Delta_{i}\right) M_{x}+\left[\begin{array}{c}
\left\|L_{x}^{1}\right\| \\
\left\|L_{x}^{1}\right\| \\
\vdots
\end{array}\right] \\
& \left(\left\|e^{A \sigma}-I\right\|\left\|x\left(k \Delta_{i}\right)\right\|+\left\|\int_{0}^{\sigma} e^{A(\sigma-\tau)} B u\left(\tau+k \Delta_{i}\right) \mathrm{d} \tau\right\|\right) \\
\leq & \alpha\left(\Delta_{i}\right) M_{x}+M_{x}\left(\max _{r} \frac{\left\|L_{x}^{r}\right\|}{M_{x}^{r}}\right) \\
& \left(\left(e^{\|A\| \sigma}-1\right)\left\|x\left(k \Delta_{i}\right)\right\|+\int_{0}^{\sigma} e^{\|A\|(\sigma-\tau)}\|B\|\left\|u\left(\tau+k \Delta_{i}\right)\right\| \mathrm{d} \tau\right)
\end{aligned}
$$

From the definitions of the bounds $X$ and $U$ and equation (A.1) we have:

$$
\left\|x\left(k \Delta_{i}\right)\right\| \leq \alpha\left(\Delta_{i}\right) X \quad \text { and } \quad\left\|u\left(\tau+k \Delta_{i}\right)\right\| \leq \alpha\left(\Delta_{i}\right) U
$$

Then:

$$
\begin{aligned}
L_{x} x(t) & \leq \alpha\left(\Delta_{i}\right) M_{x}+\alpha\left(\Delta_{i}\right) M_{x}\left(\max _{r} \frac{\left\|L_{x}^{r}\right\|}{M_{x}^{r}}\right)\left(e^{\|A\| \sigma}-1\right)\left(X+\frac{\|B\|}{\|A\|} U\right) \\
& \leq \alpha\left(\Delta_{i}\right) M_{x}\left(1+\left(e^{\|A\| \Delta_{i}}-1\right) \gamma\right)=M_{x} \frac{1+\gamma\left(e^{\|A\| \Delta_{i}}-1\right)}{1+(2 \gamma+1)\left(e^{\|A\| \Delta_{i}}-1\right)} \\
& \leq M_{x} \frac{1+\gamma\left(e^{2\|A\| \Delta_{i+1}}-1\right)}{1+(2 \gamma+1)\left(e^{2\|A\| \Delta_{i+1}}-1\right)}
\end{aligned}
$$

We show next that:

$$
\frac{1+\gamma\left(e^{2\|A\| \Delta_{i+1}}-1\right)}{1+(2 \gamma+1)\left(e^{2\|A\| \Delta_{i+1}}-1\right)} \leq \frac{1}{1+(2 \gamma+1)\left(e^{\|A\| \Delta_{i+1}}-1\right)}=\alpha\left(\Delta_{i+1}\right)
$$


for:

$$
0<\Delta_{i+1} \leq \frac{1}{\|A\|} \ln \left(\frac{\sqrt{1+4 \gamma^{2}}}{2 \gamma}\right) \Longleftrightarrow 1<e^{\|A\| \Delta_{i+1}} \leq \frac{\sqrt{1+4 \gamma^{2}}}{2 \gamma}
$$

After some manipulation, the inequality (A.5) is easily seen to be equivalent to:

$$
\gamma(2 \gamma+1) e^{2\|A\| \Delta_{i+1}}-(\gamma+1) e^{\|A\| \Delta_{i+1}}-2 \gamma^{2} \leq 0
$$

The left hand side is negative for both $e^{\|A\| \Delta_{i+1}}=1$ and $e^{\|A\| \Delta_{i+1}}=\frac{\sqrt{1+4 \gamma^{2}}}{2 \gamma}$, hence it is negative in the whole range which verifies (A.6). In (A.4) we then have that, for all $t \in\left[0, T_{f}\right]$ :

$$
L_{x} x(t) \leq \alpha\left(\Delta_{i+1}\right) M_{x} \text { for } u \in S_{\Delta_{i}}
$$

This implies, by definition, that $u \in S_{\Delta_{i+1}}$. Hence (29) follows.

\section{Proof of Lemma 6}

We consider the sequence of decreasing sampling periods $\left\{\Delta_{i}>0\right\}$ in (27). For every $\Delta_{i}$, the piecewise constant signal $u_{\Delta_{i}}(t)$ belong to $\mathcal{L}_{2}\left[0, T_{f}\right]$, because it is obtained by sampling and holding the signal $u(t)$, which belongs to $S \subseteq$ $\mathcal{L}_{2}\left[0, T_{f}\right]$. Thus, the sequence of functions $\left\{u_{\Delta_{i}}(t)\right\}$ converges point-wise almost everywhere (i.e. except on a set of zero measure) to $u(t)$. By Theorem 1.6 in [11], this implies that $\left\{u_{\Delta_{n}}(t)\right\}$ converges to $u(t)$ in an $\mathcal{L}_{2}$ sense, i.e.:

$$
\lim _{i \rightarrow \infty}\left\|u-u_{\Delta_{i}}\right\|_{2}=\lim _{\Delta_{i} \rightarrow 0}\left\|u-u_{\Delta_{i}}\right\|_{2}=0
$$

This gives the result. 
We consider the Hilbert spaces $\mathcal{V}=\mathcal{L}_{2}\left(0, T_{f} ; \mathbb{R}^{m}\right)$ and $\mathcal{Z}=\mathbb{R}^{n} \times \mathcal{L}_{2}\left(0, T_{f} ; \mathbb{R}^{n}\right)$, with inner products:

$$
\begin{gathered}
\left\langle f_{1}, f_{2}\right\rangle_{\mathcal{V}}=\int_{0}^{T_{f}} f_{1}^{T}(t) f_{2}(t) \mathrm{d} t \quad ; f_{1}, f_{2} \in \mathcal{V} \\
\left\langle g_{1}, g_{2}\right\rangle_{\mathcal{Z}}=\left(g_{1}^{0}\right)^{T} g_{2}^{0}+\int_{0}^{T_{f}}\left(g_{1}^{1}(t)\right)^{T} g_{2}^{1}(t) \mathrm{d} t \\
; g_{1}=\left[\begin{array}{c}
g_{1}^{0} \\
g_{1}^{1}(t)
\end{array}\right], g_{2}=\left[\begin{array}{c}
g_{2}^{0} \\
g_{2}^{1}(t)
\end{array}\right] \in \mathcal{Z}
\end{gathered}
$$

We rewrite the response signal as:

$$
z=\left[\begin{array}{c}
z^{0} \\
z^{1}(t)
\end{array}\right]=\left[\begin{array}{c}
P^{1 / 2} x\left(T_{f}\right) \\
Q^{1 / 2} x(t)
\end{array}\right]=\left[\begin{array}{c}
P^{1 / 2} \int_{0}^{T_{f}} e^{A\left(T_{f}-\xi\right)} B u(\xi) d \xi \\
Q^{1 / 2} \int_{0}^{t} e^{A(t-\xi)} B u(\xi) d \xi
\end{array}\right] \in \mathcal{Z}
$$

Note that this formulation is similar to that employed in [10].

The cost function (3) and the system dynamics can then be expressed, respectively, in the form:

$$
\begin{aligned}
J & =\left\|R^{1 / 2} u\right\|_{\mathcal{V}}^{2}+\|z\|_{\mathcal{Z}}^{2} \\
z & =\mathcal{F} x_{o}+\mathcal{G} u
\end{aligned}
$$

where $\mathcal{F}$ and $\mathcal{G}$ are linear operators defined by:

$$
\begin{aligned}
\mathcal{F} x_{o} & =\left[\begin{array}{c}
\left(\mathcal{F}_{\Delta} x_{o}\right)^{0} \\
\left(\mathcal{F}_{\Delta} x_{o}\right)^{1}(t)
\end{array}\right]=\left[\begin{array}{c}
P^{1 / 2} e^{A T_{f}} x_{o} \\
Q^{1 / 2} e^{A t} x_{o}
\end{array}\right] \quad ; t \in\left[0, T_{f}\right], x_{o} \in \mathbb{R}^{n} \\
\mathcal{G} u & =\left[\begin{array}{c}
(\mathcal{G} u)^{0} \\
(\mathcal{G} u)^{1}(t)
\end{array}\right]=\left[\begin{array}{c}
P^{1 / 2} \int_{0}^{T_{f}} e^{A\left(T_{f}-\xi\right)} B u(\xi) d \xi \\
Q^{1 / 2} \int_{0}^{t} e^{A(t-\xi)} B u(\xi) d \xi
\end{array}\right] ; t \in\left[0, T_{f}\right], u \in \mathcal{V}
\end{aligned}
$$

We can then rewrite (A.13) as:

$$
J=J(\tilde{u})=J_{o p t}+\left\langle\tilde{u},\left(R+\mathcal{G}^{*} \mathcal{G}\right) \tilde{u}\right\rangle
$$


where:

$$
\begin{aligned}
\tilde{u} & =u-u_{\text {opt }} \\
u_{\text {opt }} & =-\left(R+\mathcal{G}^{*} \mathcal{G}\right)^{-1} \mathcal{G}^{*} \mathcal{F} x_{o} \\
J_{\text {opt }} & =x_{o}^{T} \mathcal{F}^{*}\left(I+\mathcal{G} R^{-1} \mathcal{G}^{*}\right)^{-1} \mathcal{F} x_{o}
\end{aligned}
$$

We note that $u_{\text {opt }}$ and $J_{\text {opt }}$ are the unconstrained optimal control signal and cost.

The operator $R+\mathcal{G}^{*} \mathcal{G}$ in (A.17) is a compact bounded self-adjoint operator. It can thus be expressed as:

$$
R+\mathcal{G}^{*} \mathcal{G}=\mathcal{S}^{*} \mathcal{S}
$$

We then have that, for $u_{1}, u_{2} \in \mathcal{L}_{2}\left[0, T_{f}\right]$ :

$$
\begin{aligned}
\left|J\left(u_{1}\right)-J\left(u_{2}\right)\right| & =\left|\left\langle\tilde{u}_{1}, \mathcal{S}^{*} \mathcal{S} \tilde{u}_{1}\right\rangle-\left\langle\tilde{u}_{2}, \mathcal{S}{ }^{*} \mathcal{S} \tilde{u}_{2}\right\rangle\right| \\
& =\left|\left\langle\mathcal{S} \tilde{u}_{1}, \mathcal{S} \tilde{u}_{1}\right\rangle-\left\langle\mathcal{S} \tilde{u}_{2}, \mathcal{S} \tilde{u}_{2}\right\rangle\right| \\
& =\left|\left\|\mathcal{S} \tilde{u}_{1}\right\|^{2}-\left\|\mathcal{S} \tilde{u}_{2}\right\|^{2}\right| \leq\left\|\mathcal{S} \tilde{u}_{1}-\mathcal{S} \tilde{u}_{2}\right\|^{2} \\
& \leq\|\mathcal{S}\|^{2}\left\|\tilde{u}_{1}-\tilde{u}_{2}\right\|^{2} \leq \sigma_{\max }\left\|u_{1}-u_{2}\right\|^{2}
\end{aligned}
$$

where $\sigma_{\max }>0$ is the largest singular value of the operator $\mathcal{S}$.

Lemma 7 then follows by taking $u_{2}=u^{*}, \delta_{\varepsilon}=\sqrt{\varepsilon / \sigma_{\max }}$, and on recalling that $u^{*}$ is the optimal control signal, so $J(u) \geq J\left(u^{*}\right)$ for all $u$.

\section{References}

[1] K.J. Åström and B. Wittenmark. Computer Controlled Systems. Theory and Design. Prentice Hall, Englewood Cliffs, N.J., 3rd edition, 1997.

[2] L.D. Berkovitz. Optimal Control Theory. Springer-Verlag, New York, 1974.

[3] M. Cannon and B. Kouvaritakis. Infinite horizon predictive control of constrained continuous time linear systems. Automatica, 36:943-955, 2000.

[4] M. Cannon, B. Kouvaritakis, and J.A. Rossiter. Efficient active set optimization in triple mode mpc. IEEE Transactions on Automatic Control, 46(8):1307-1312, 2001.

[5] X. Cheng and B.H. Krogh. Stability-constrained model predictive control. IEEE Transactions on Automatic Control, 46(11):1816-1820, 2001. 
[6] D. Chmielewski and V. Manousiouthakis. On constrained infinite-time linear quadratic optimal control. Systems and Control Letters, 29:121-129, 1996.

[7] S.L. de Oliveira Kothare and M. Morari. Contractive model predictive control for constrained nonlinear systems. IEEE Transactions on Automatic Control, 45(6):1053-1071, 2000.

[8] A. Feuer and G.C. Goodwin. Sampling in Digital Signal Processing and Control. Birkhäuser, Boston, 1996.

[9] G.C. Goodwin, M.M. Serón, and J. De Doná. Constrained Control and Estimation. Springer-Verlag, 2004.

[10] A. Kojima and M. Morari. LQ control for constrained continuous-time systems: an approach based on singular value decomposition. In Proc. of the 40th IEEE Conference on Decision and Control, 5:4838-4844, 2001.

[11] S. Lang. Real and Functional Analysis. Springer-Verlag, 3rd edition, 1993.

[12] D.Q. Mayne, J.B. Rawlings, C.V. Rao, and P.O.M.Scokaert. Constrained model predictive control: Stability and optimality. Automatica, 36:789-814, 2000.

[13] R.H. Middleton and G.C. Goodwin. Digital Control and Estimation. A Unified Approach. Prentice Hall, Englewood Cliffs, New Jersey, 1990.

[14] D.E. Miller and M. Rossi. Simultaneous stabilization with near optimal LQR performance. IEEE Transactions on Automatic Control, 46(10):1543-1555, October 2001.

[15] J.B. Rawlings and K.R. Muske. The stability of constrained receding-horizon control. IEEE Transactions on Automatic Control, 38(10):1512-1516, 1993.

[16] P.O.M. Scokaert and J.B. Rawlings. Constrained linear quadratic regulation. IEEE Transactions on Automatic Control, 43(8):1163-1169, 1998.

[17] M. Sznaier and M.J. Damborg. Suboptimal control of linear systems with state and control inequality constraints. In Proc.of the 26th IEEE Conference on Decision and Control, pages 761-762, Los Angeles, USA, 1987.

[18] C. Van De Panne. Methods for Linear and Quadratic Programming. American Elsevier Pub. Co., New York, 1975.

[19] R. Vinter. Optimal Control. Birkhäuser, Boston, 2000. 